\title{
Decision interaction between auditory channels
}

\author{
R. D. SORKIN, L. D. POHLMANN, and D. D. WOODS \\ Purdue University, West Lafayette, Indiana 47907
}

\begin{abstract}
Observers detected signals presented in a two-channel, simultaneous detection task at 630 and $1,400 \mathrm{~Hz}$. The familiar pattern of interference between the frequency channels was observed: detectability in one channel was depressed on trials when a signal or a yes response occurred in the other channel. On each trial, measures were made of the energy within specified frequency bands around each signal frequency. The relationships between these measures and performance in each channel were studied. There was no consistent relationship between the magnitude of the measure in one channel and performance in the other. Interactions between the channels probably originate in the response process of the interfering channel.
\end{abstract}

The nature of the interaction between inputs which are presented simultaneously in different spectral regions remains a question of major interest to acoustical scientists. A general experimental question has concerned the magnitude of the performance decrement which occurs in such tasks. The many types of experiments which have been employed to study these effects include (a) the detection of spectrally complex signals in noise and (b) the effects of various types of sinusoidal or noise maskers on signal detection. We have reported a series of experiments which employ a different procedure, using a shared attention or two-channel paradigm (Sorkin, Pastore, \& Pohlmann, 1972; Sorkin, Pohlmann, \& Gilliom, 1973). In these experiments, the observer must simultaneously perform two independent signal-detection tasks; the signals to be detected are confined to specified frequency regions or earphone channels. We have compared the detectability of each channel in these tasks to detectability in comparable singlechannel tasks in which signals occurred only in one specified channel. Similar experiments with timepaced pure-tone signals (Moray, Fitter, Ostry, Favreau, \& Nagy, 1976), alphanumeric signals (Ostry, Moray, \& Marks, Note 1), and acoustical and visual signals (Shiffrin \& Grantham, 1974) have been reported in the recent literature. In general, these studies have confirmed the presence of the specific cross-channel effects reported by our laboratory (Sorkin et al., 1972). There is usually a small decrement in performance in the two-channel task compared with the single-channel task. However, the detectability of each channel in the twochannel task is critically dependent on the nature of trial events in the other channel. Performance on trials when neither a signal nor a yes response has

This research was supported by a National Science Foundation grant to the first author. L. D. Pohlman's current address: Department of Psychology, Arizona State University, Tempe, Arizona 85281. occurred in the irrelevant channel is essentially identical to single-channel performance. Detectability when either a signal or a yes response has occurred in the remote channel is from $5 \%$ to $50 \%$ lower than that when neither of those events has occurred. The fact that this interaction obtains even when the channels are isolated by earphone, frequency, or sensory modality supports the argument that the locus of interaction is at a level in the nervous system higher than that involved in traditional sensory masking experiments.

One interpretation of the interference effect is that an observer has no difficulty in monitoring two or even three spectrally isolated regions, so long as a large-magnitude observation, e.g., one leading to a yes response, does not occur at any of those regions. Indeed, in an experiment requiring the simultaneous detection of signals in three separated frequency regions, we have found similar results (Pohlmann \& Sorkin, 1974). These data are suggestive of some recent results reported by Ahumada, Marken, \& Sandusky (1975). In their experiments, the observer seems to be monitoring the outputs of a bank of narrow filters (we will neglect the interesting temporal effects which they report) and basing his responses on the entire pattern of filter outputs: if any of the filter outputs has reached some critical value, the observer responds "yes"; if none have reached some (other) value, he responds "no." The notion is that the observer bases his responses on an analyzed pattern of filter outputs, rather than on a simpler singlefilter strategy. This is consistent with the interesting result (Ahumada, Note 2) that the correlation of the human observer's responses with that of a single-filter model differs on signal and no-signal trials. Our interest in the Ahumada experiments is the apparent similarity to our results in the two-channel detection of two or three frequencies. Multichannel detection or shared attention exists without any decrement in performance, so long as no signal-like event occurs on any of the 
channels. We decided to study the correlation between particular physical properties of the stimulus with the interactions between channels observable in the two-channel task.

An obvious hypothesis concerning this interaction is that the detectability in one channel should be a decreasing function of the magnitude of the energy detected in the other channel. Large observations on a channel should be associated with trials when that channel contained a signal or a yes response. The hypothesis is thus consistent with our previously discussed interrupt model of interference, wherein the presence of a signal-like observation in one channel causes a disruption of processing in the other (Gilliom \& Sorkin, 1974; Sorkin \& Pohlmann, 1973). It is also consistent with a general masking-like interpretation, in that the magnitude of the observation, e.g., the presence of signal-relevant energy in one channel, causes interference with the other channel. That is, (a) there is an effective leakage of stimulation into the listening band at the other frequency, or (b) the external level in one channel drives the system gain down in the other, thereby increasing the effect of internal noise. Many other specific mechanisms could be cited. For example, the magnitude of the observation in one channel could be related to the response criterion in the other, either to cause some consistent shift in criterion or to increase criterion variability. In the former case, the shift would be apparent; in the latter case, overall detectability would be inversely related to the measure.

In order to evaluate this interaction, we recorded the actual noisy inputs (plus synchronization signals) to the observer's headphone during an extensive series of two-channel tasks. This information was later processed to give trial-by-trial measures of the energy in a narrow band around each of the signal frequencies. If the first hypothesis was correct, the magnitude of the energy measures in one frequency channel should be negatively correlated with performance in the other. ${ }^{1}$

A more complex hypothesis is that performance is a U-shaped function of the energy-derived measure in the remote channel. Such an effect could be justified on the following basis: midrange observations on a channel reflect maximum uncertainty about the existence of signal or noise in that channel, in that they are nearest to the response criterion for the channel. Those midrange observations constitute maximum uncertainty for the observer in making a response to that channel, and that uncertainty will influence and interfere with processing in the other channel. Perhaps that interference will be due to some competition for processing resources which are necessary for final decision and response. One might say that trial difficulty was the controlling variable in the cross-channel interaction.

Another general hypothesis is that there will be no correlation between observable events or performance in one channel and the computed signal-relevant energy in the other channel. This hypothesis implies a locus of channel interaction which is removed from the early processing stages. The decision measure for a channel will of necessity be a monotonic function of the signal-relevant energy in that channel. Any interference at that stage must come from sources that are uncortelated with that measure, such as independent internal noise sources either in the peripheral system or higher up in the nervous system. However, there is an empirical result which also must be reconciled with this hypothesis: Performance is clearly impaired when a signal or a yes response has occurred in the interfering channel. Since the hypothesis precludes a signal-caused locus of interference, the remaining alternative is interference induced by the existence of a particular response (yes) in the irrelevant channel. Gilliom and Mills (1974) have reported crosschannel interference between channels in which a "signal" response is required by a gap in a sinusoid signal (rather than a pulse), a result which is consistent with this hypothesis. We hoped to evaluate these hypotheses in an appropriate twochannel experiment, wherein trial-by-trial measures were made of the signal-relevant energy in each channel.

\section{PROCEDURE}

Two female students with normal hearing served as paid observers. They were housed in a single-walled Industrial Acoustics chamber inside an acoustically insulated room. The basic task was a single-interval yes-no task; trial events were controlled and recorded by a PDP $-8 / \mathrm{L}$ computer. Five hundred trials were run each day in 100 -trial blocks for about 10 working days.

On each trial, the observer was to make two separate yes/no responses to the possible occurrence of two sinusoid signals, one at $630 \mathrm{~Hz}$ and one at $1,400 \mathrm{~Hz}$. The signals were $100 \mathrm{msec}$ in duration and could occur only in the same visually defined 100 -msec observation interval. The signals were independently presented with a probability of occurrence of 0.5 . The observer had separate yes/no buttons for each frequency signal. Continuous broad-band Gaussian noise was present in the headphone (at an overall level of $62 \mathrm{~dB}$ SPL); the signals and noise were monaural. Signals were gated on and off at positive zero crossings, at $E / N_{0}$ levels of 13.2 and 17.0 , respectively, for the 630 - and $1,400-\mathrm{Hz}$ signals.

During the course of the experiment, the actual earphone inputs were recorded on one channel of audio tape, with synchronization signals recorded on a second channel to mark the precise time of the observation intervals. These recorded observations were subsequently processed in the following way: The observations were filtered by analog means at each signal frequency and with either a narrow-band $(50-\mathrm{Hz}$ passband centered on 630 or $1,400 \mathrm{~Hz}$ using a General Radio wave analyzer, $1,900-\mathrm{A}, 30-\mathrm{dB}$ attenuation at $\pm 100 \mathrm{~Hz}, 60 \mathrm{~dB}$ at 
Figure 1. Distribution of integrated output (in arbitrary units) of narrow-band filter for 400 trials. EM/N and EM/S + N represent, respectively, energy messure given noise alone and energy measure given signal plus noise. The partitions along the abscissa represent approximately equal numbers of trials when low output, uncertain output, and high output measures resulted.

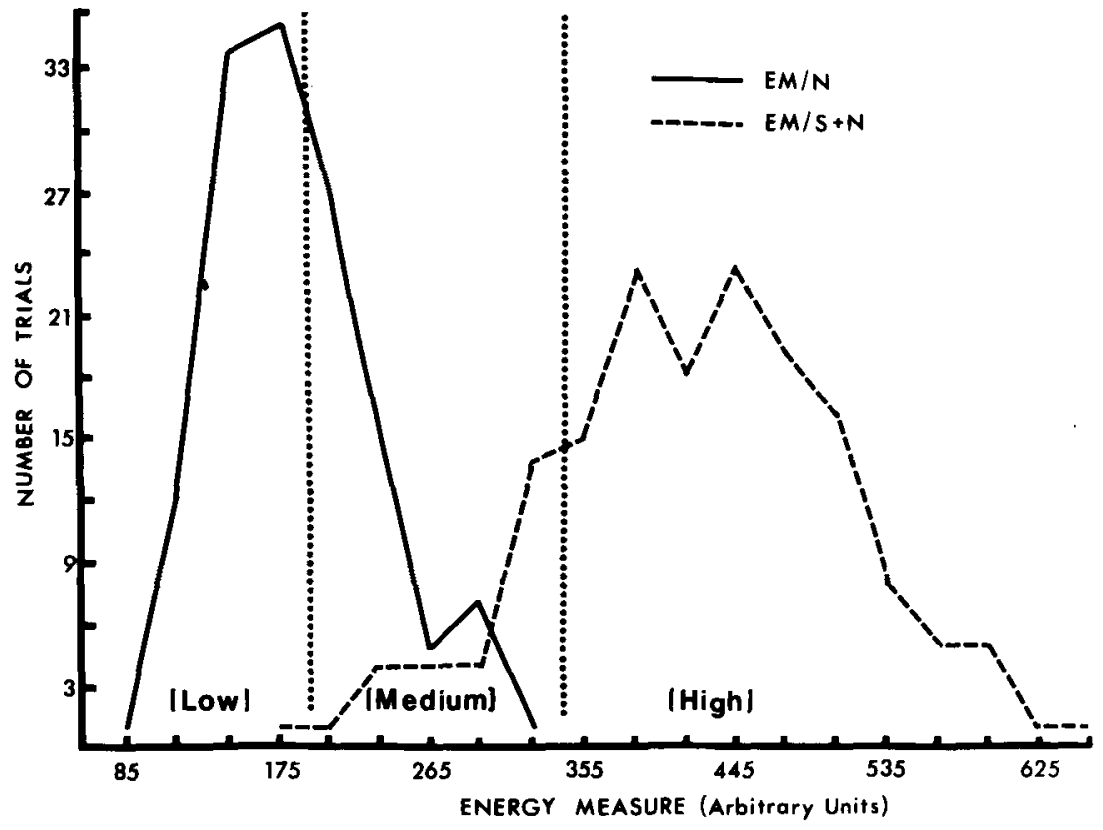

$\pm 250 \mathrm{~Hz}$ ) or medium-width ( $220 \mathrm{~Hz}$ passband centered on 630 or $1,400 \mathrm{~Hz}$ using $>60 \mathrm{~dB}$ per octave attenuation) filter. These filter outputs were digitized (10-bit analog-to-digital converter at approximately 10,000 samples $/ \mathrm{sec}$ ), and the negative-polarity sample values were summed over the duration of the observation interval. Thus, for every trial, four relative measures of the channel energies were computed, based on the sampled, half-wave rectified, and integrated outputs of the narrow and medium-wide filters at each signal frequency.

\section{RESULTS AND DISCUSSION}

The magnitude of the energy measure based on narrow-band filtering was highly correlated (typical value for a session, $r=0.85$ ) with the occurrence of signal in the appropriate channel. The resolving power of the energy measure can be assessed by assuming a decision criterion on the measure and partitioning each trial into a "yes" or "no" response. Hits and false alarms can then be determined and a d' computed. Assuming a response criterion in the middle range of the measure, a detectability of 3.0 or higher resulted from such a calculation based on the narrow-band filter energy measure. ${ }^{2}$ The measures based on broadband filtering were somewhat less correlated $(r=$ 0.52 ) with signal occurrence in the appropriate channel. There was no significant correlation between the measures in one channel and the occurrence of signal in the other; a typical $r$ was 0.03 . Figure 1 shows the distribution of the narrow-band measure (at $630 \mathrm{~Hz}$ ) for 400 trials. High-energy measures in a channel correlated highly with yes responses in that channel; 0.61 was a typical value for the narrow, 0.40 for the broad filter.

The previously reported dependence of detect- ability on signal and response events in the other channel was replicated. Table 1 is a summary of the data. The pattern of dependence of $d^{\prime}$ on events in the other channel followed those reported in our previous experiments (Sorkin et al., 1972; Sorkin et al. 1973). The observers' criterion, $\beta$, also seemed to depend on signal or response events in the other channel. Note that the measure of $\beta$ we have employed is not the traditional ratio of ordinates of the signal and noise distributions at the criterion point. For computational convenience, we have defined $\beta$ as the distance from the signal mean to the criterion point, divided by the distance between the means.

Of primary concern was the relationship between the magnitude of the energy measure in one channel and performance in the other. Two general approaches to assessing this relation were used. In the first approach, we partitioned the experimental trials for a day's session into three classes according to the magnitude of the energy measure in a particular channel. The energy measure continuum was seg-

Table 1

Average Detectabilty and Criterion for Exch Oberver and Channed

\begin{tabular}{|c|c|c|c|c|}
\hline & \multicolumn{2}{|c|}{ Observer 1} & \multicolumn{2}{|c|}{ Observer 2} \\
\hline & 630 & 1400 & 630 & 1400 \\
\hline$d_{i}^{\prime}$ & $1.900(.390)$ & $1.912(.537)$ & $1.979(.453)$ & $1.948(.473)$ \\
\hline $\begin{array}{l}\mathrm{d}_{\mathrm{i}} / \mathrm{S}_{\mathrm{j}} \\
\mathrm{d}_{\mathrm{i}} / \mathrm{N}_{\mathrm{j}} \\
\% \text { difference }\end{array}$ & $\begin{array}{l}1.829(.363) \\
2.000(.421) \\
9.4\end{array}$ & $\begin{array}{l}1.869(.492) \\
2.087(.589) \\
11.7\end{array}$ & $\begin{array}{l}1.944(.430) \\
2.046(.480) \\
5.2\end{array}$ & $\begin{array}{l}1.878(.499) \\
2.078(.440) \\
10.7\end{array}$ \\
\hline $\begin{array}{l}\mathrm{d}_{\mathrm{i}} / \text { Yes; } \\
\mathrm{d}_{\mathrm{i}} / \mathrm{No} ; \\
\% \text { difference }\end{array}$ & $\begin{array}{l}1.825(.315) \\
2.013(.486) \\
10.3\end{array}$ & $\begin{array}{l}1.879(.421) \\
2.078(.618) \\
10.6\end{array}$ & $\begin{array}{l}1.960(.435) \\
2.033(.476) \\
3.7\end{array}$ & $\begin{array}{l}1.867(.487) \\
2.085(.457) \\
11.7\end{array}$ \\
\hline
\end{tabular}

Note- $\beta$ values are shown in parentheses. All entries are based on 5 days at 500 trials per day. 
Table 2

Average Detectability and Criterion on Trials When the Energy Measure in the Other Channel was in Low, Medium, or High Range

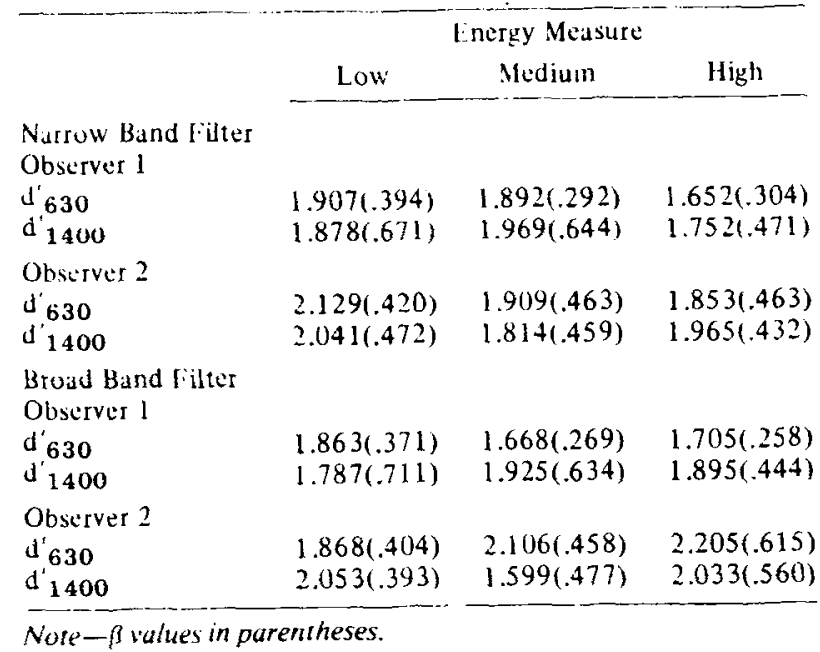

Table 3

Correlation ( $r_{p b}$ ) of Energy Measure in One Channel with Yes or No Response in the Other Channel

Observer $1 \quad$ Observer 2

Day Channel $\quad r_{p b}\left(d^{\prime} / N_{j}\right)-\left(d_{i}^{\prime} / S_{j}\right) r_{p b}\left(d_{i}^{\prime} / N_{j}\right)-\left(d_{i} / S_{j}\right)$

\begin{tabular}{ccrrrr}
\hline & Narrow Bund Filter & & & & \\
1 & 630 & -.231 & .137 & -.053 & -.028 \\
& 1400 & -.055 & .235 & -.040 & .017 \\
2 & 6311 & -.084 & .198 & -.010 & .123 \\
& 1400 & -.080 & .003 & -.060 & .230 \\
3 & 630 & -.130 & .217 & -.011 & .444 \\
& 1400 & -.126 & -.229 & -.080 & -.161 \\
4 & 630 & -.043 & .229 & -.028 & -.032 \\
& 1400 & -.039 & .238 & -.122 & .074 \\
5 & 630 & -.117 & -.023 & .022 & .004 \\
& 1400 & -.015 & .496 & -.006 & .843 \\
Broad Band Filter & & & & & \\
1 & 630 & -.156 & .137 & -.050 & -.028 \\
& 1400 & -.050 & .235 & .007 & .017 \\
\multirow{2}{*}{2} & 630 & -.078 & .198 & -.035 & .123 \\
& 1400 & -.002 & .003 & -.033 & .230 \\
3 & 630 & -.037 & .217 & -.018 & .444 \\
& 1400 & -.102 & -.229 & -.094 & -.161
\end{tabular}

Note - The conditional performance decrement (bused on signat or noise in the other channel) is also tabled.

mented so that the classes contained approximately equal numbers of trials. The vertical lines in Figure 1 depict a typical partition. Performance $\left(d^{\prime}, \beta\right)$ for the other channel was then computed for the trials in each class separately. This was done for both channels. No consistent pattern of dependence (either overall, or within a day's run) of $d^{\prime}$ or $\beta$ on the nature of the trial measure (high, medium, or low) in the other channel was found. Table 2 summarizes these data.

The second analysis involved computation of the point biserial correlation between the original energy measure in one channel and either the response (yes or no) or the outcome (correct or incorrect) in the other channel. Table 3 shows the correlations computed on each day for yes-no responses, and Table 4 for correct or incorrect responses. Note that the value of a statistically significant correlation for a block of 500 trials is quite small, $r=0.09$ for $p<.05$. A correlation of 0.10 would be much less than that reasonably expected to account for the cross-channel decrements observed. Less than half of the correlations reach significance in the proper direction. On several days when the cortelation is significant, the conditional performance decrement $\left(d^{\prime}{ }_{i} /\right.$ Noise $_{j}-d^{\prime}{ }_{i} /$ Signal $\left._{j}\right)$ is either low or in the wrong direction.

It is possible that a dependence between the energy measure and performance could exist, if the region of dependence were very small and its location unspecified, such as near the observer's actual response criterion in that channel. If such a dependence existed, and if this uncertainty region were biased to include more yes than no responses, the observable pattern of dependencies could certainly result. There was no way to evaluate this possibility in the present study. It might be interesting to run a two-channel detection experiment in which the observer was required to make rating responses to each channel. In that case, one would be able to approximate the actual observer decision value for a channel on each trial, and to determine the relationship between performance in each channel and that measure. The use of a rating task may add to

Table 4

Correlation ( $r_{p b}$ ) of Energy Measure in One Channel with Correct or Incorrect Response in the Other Channe!

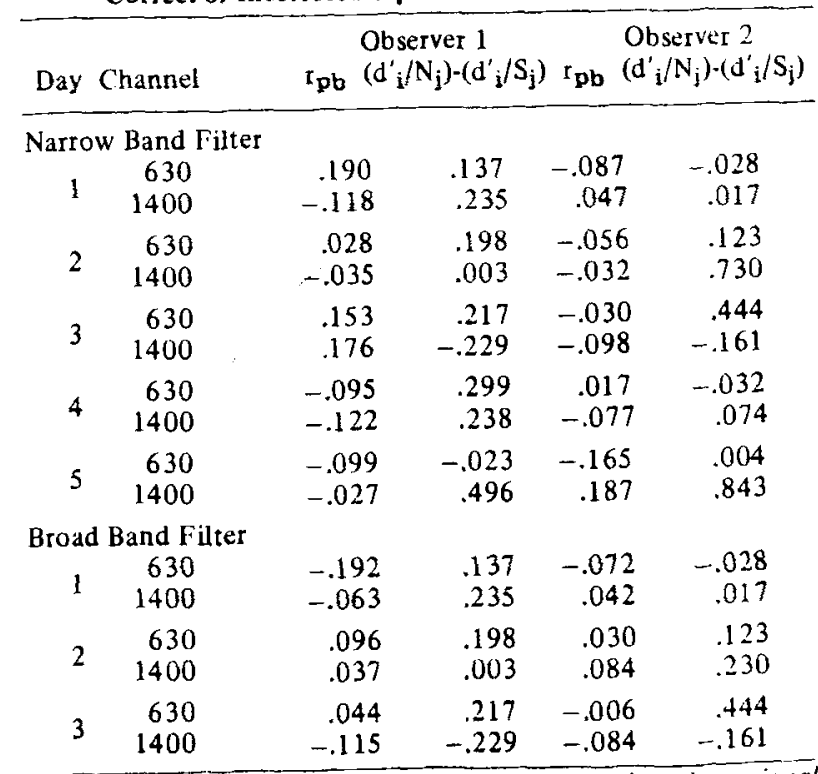

Nole-The conditional performance decrement (based on signal or noise in the other channel) is also tabled. 
interference between the channels, if criterion variability is involved in the interaction. McNicol (1975) has demonstrated the effects of noise on both the sensory and the decision processes. He suggested that tasks requiring multiple responses, such as rating tasks, must tolerate reduced performance compared to yes/no and forced-choice procedures, because the requirement to hold several simultaneous criteria adds noise to the observer's decision process. We note that this increased criterion variability may also obtain in multichannel detection, where more than one response criterion is required by the task. This is another possible explanation for the performance decrement in shared attention tasks, but it does not describe the kinds of dependencies observed.

The absence of any observable correlation between the energy-related measures in one channel and performance in the other, argues strongly against either of the first two hypotheses proposed. The presence of an effect that was dependent on the level of input energy at the signal frequency would have been satisfying in its simplicity. In fact, there is other evidence consistent with the alternative view, that the interference is not related to the input energy in the other channel. We have observed little reduction in the magnitude of the cross-channel interference when the signals were isolated by earphone channel (with independent noise, $\mathrm{Nu}$ ) or by slightly offsetting the observation interval in time (Gilliom \& Sorkin, 1974). Thus this interaction occurs almost unattenuated by spatial, temporal, and spectral isolation. The locus of the interaction must be beyond the level of the peripheral system, and must not be correlated with the magnitude of the observation made in the interfering channel.

It is possible that the magnitude of independently generated internal noise in the first stages of the system is directly related to the cross-channel decrement in performance, but this seems a remote possibility. First, such an effect ought to result in a correlation between correct events in both channels, a "state" correlation, in Garner and Morton's (1969) terms, which we have not observed in our recent two-channel experiments. Secondly, there should be a reversal in the dependence on a yes response in the other channel when signal gaps or decrements, rather than pulses or increments, are identified as signals. That result has not been observed (Gilliom \& Mills, 1974). It seems very unlikely that noise in a stage preceding computation of the decision statistic and uncorrelated with the external input (either noise or signal) should have an influence on detectability in the other channel. One way such a state correlation could occur is if there is an internal noise source which is common to both channels; however, in that case we would expect to see a dependency between the computed criterion in one channel on trials when certain response events had occurred in the other. That result would also obtain if some criterion noise source was common to both the channels; in that case, the dependence between specific responses in each channel also would be apparent. These latter cases are interesting in spite of not being relevant to the auditory detection situation, because the outcomes are similar to those predicted by response interference models (Kantowitz, 1973). Rather, the result in the present experiment suggests channel interactions which are occurring somewhere beyond the initial stages of signal processing, and not simply involving tendencies to make identical responses in each channel.

This view is consistent with some hypotheses about channel interaction discussed by Shiffrin and Grantham (1974), who argue that memory and decision factors subsequent to perceptual processing are involved in limitations on multichannel processing. In the terminology of Norman and Bobrow (1975), the cross-channel interaction may reflect limitations in processing resources such as sampling rate, effort, certain types of memory capacity, and output processes; or they may reflect limitations in certain input ("data") processes which are not a function of the external signal-to-noise ratio, such as memory for channel frequency or waveform. The latter category can probably be safely ruled out in most two-channel experiments where it is unlikely that confusion will exist between the channels. What remains is an effect involving the allocation of processing resources in one channel as a function of particular responses in the other channel. In Garner's terms, the interaction is a type "d" process. wherein one response system has an effect on the processing of information in the other channel. Kantowitz (1973) has reported convincing evidence for interference between the response processes in a reaction time task. There are many stages where a channel response process might have its effect on detection in the other channel. The sampling rate could be reduced, or the internal noise or criterion variability increased, by response processes in the remote channel. Exactly how these effects might proceed over time remains an open question.

\section{REFERENCE NOTES}

1. Ostry, D., Moray, N., \& Marks, G. Attention, practice, and semantic targets. Unpublished manuscript, 1975.

2. Ahumada, A. Detection of tones masked by noise: A comparison of human observers with digital-computer-simulated energy detectors of varying bandwidths. Technical Report No. 29. UCLA Department of Psychology Human Communication Lab., March 1967. 


\section{REFERENCES}

Ahumada, A., Marken, R., \& Sandusky, A. Time and frequency analyses of auditory signal detection. Journal of the Acoustical Society of America, 1975, 57, 385-390.

GARNER, W. R. The processing of information and structure. Potomac, Md: Erlbaum Associates, 1974.

Garner, W. R., \& Morton, J. Perceptual independence: Definitions. models, and experimental paradigms. Psychological Bulletin. 1969, 72, 233-259.

Gilliom. J. D., \& Mirls, W. M. Two-channel detection of the missing event. Journal of the Acoustical Society of America, 1974, 55, 519. (Abstract)

Gilliom. J. D., \& Sorkin, R. D. Sequential vs. simultaneous two-channel signal detection: More evidence for a high-level interrupt theory. Journal of the Acoustical Society of America, 1974, 56, 157-164.

KANTOWITZ, B. H. Response force as an indicant of conflict in double stimulation. Journal of Experimental Psychology, 1973, 100. 302-309.

McNicol, D. Feedback as a source of information and as a source of noise in absolute judgments of loudness. Journal of Experimental Psychology: Human Perception and Pertormance, 1975. 104. 175-182.

Moray, N.. Fitter, M., Ostry, D., Favreau, D., \& Nagy, V. Attention to pure tones. Quarterly Journal of Experimental Psychology. 1976, in press.

Norman, D. B., \& Bobrow. D. G. On data-limited and resource-limited processes. Cognitive Psychology, 1975, 7. 44-64.

Pohlmann, L. D., \& Sorkin, R. D. Simultaneous threechannel signal detection. Journal of the Acoustical Society of America, 1974, 55. 519. (A bstract)
Shiffrin, R. M., \& Grantham, D. W. Can attention be allocated to sensory modalities? Perception \& Psychophysics. $1974,15,460-474$.

Sorkin, R. D., Pastore, R. E., \& Pohlmann, L. D. Simultaneous two-channel signal detection. II. Correlated and uncorrelated signals. Joumal of the Acoustical Society of America, 1972, 51, 1960-1965.

Sorkin, R. D., \& Pohlmann, L. D. Some models of observer behavior in two-channel auditory signal detection. Perception \& Psychophysics, 1973, 14, 101-109.

Sorkin, R. D., Pohlmann, L. D., \& Gilliom, J. D. Simultaneous two-channel signal detection. III. 630 and $1400 \mathrm{~Hz}$ signals. Journal of the Acoustical Society of America, 1973, 53, 1045-1050.

\section{NOTES}

1. A reviewer has suggested another related hypothesis: the detectability in one channel could be positively correlated with the magnitude of the observation in the other channel. If a large-magnitude observation (e.g., during integration) allowed a more rapid decision, processing capacity could be released for the other channel.

2. The reader will note that these detectabilities are much lower than the ideal $d$ 's that may be computed from the $\mathrm{E} / \mathrm{N}_{\mathrm{O}}$ levels, but much higher than those for the human observers.
(Received for publication June 1975: revision received January 20.1976 .) 